\title{
Vehicular VLC Frequency Domain Channel Sounding and Characterization
}

\author{
Bugra Turan, Gokhan Gurbilek, Ali Uyrus and Sinem Coleri Ergen \\ Department of Electrical and Electronics Engineering, Koc University, Sariyer, Istanbul, Turkey, 34450 \\ E-mail: bturan14@ku.edu.tr, ggurbilek13@ku.edu.tr, auyrus@ieee.org, sergen@ku.edu.tr
}

\begin{abstract}
Vehicular visible light communications (V2LC) has recently gained popularity as a complementary technology to radio frequency (RF) based vehicular communication schemes due to its low-cost, secure and RF-interference free nature. In this paper, we propose outdoor vehicular visible light communications (V2LC) frequency domain channel sounding based channel model characterization under night, sunset and sun conditions with the usage of vector network analyzer (VNA) and commercial offthe-shelf (COTS) automotive light emitting diode (LED) light. We further bring forward a new practical system bandwidth criteria named as effective usable bandwidth (EUB) for an endto-end V2LC system with respect to real world measurements. We demonstrate outdoor static V2LC channel measurement results, taking into account vehicle light emitting diode (LED) response, road reflections from nearby vehicles and various day light conditions with respect to varying inter-vehicular distances. Measurement results indicate that, sun light decreases system effective usable bandwidth due to the limited dynamic range of avalanche photodiode (APD), nearby vehicles cause constructive interference whereas road reflections change time dispersion characteristics of the V2LC channel.

Index Terms-Vehicular communication, visible light communication, vehicle to vehicle communication, VLC Channel Sounding, VLC Channel Model Development
\end{abstract}

\section{INTRODUCTION}

VLC using modulated optical radiation of LEDs without any noticeable effect on human eye, is considered to be a promising optical wireless communication technology. Utilizing base-band transmission with intensity modulation / direct detection (IM/DD), VLC is also considered to be lowcomplexity and low cost solution when compared to radio frequency (RF) based wireless communication alternatives. Furthermore, VLC is gaining popularity as it provides secure communications due to line-of-sight $(\mathrm{LoS})$ requirement with its RF and electromagnetic interference free nature. VLC usage is foreseen for various applications, including high speed indoor communications [1], underwater communications [2], intelligent transportation systems and vehicular communications [3]. As a prominent application, V2LC is foreseen to be a complementary solution for vehicle to everything communications (V2X) applications. As RF based 802.11p/DSRC and Cellular-V2X communication schemes are targeting utilization of the limited Intelligent Transportation Systems (ITS) 5.9 Ghz band, spectrum scarcity and RF interference issues should be addressed for reliable V2X. On the other hand, autonomous and cooperative driving applications are envisaged to require more advanced driver-assistance systems (ADAS) sensor information sharing capabilities from nearby vehicles. Hence, V2LC is foreseen to be a strong candidate for offloading RF vehicular networks considering LoS dense traffic (i.e. stop and go) and platoon usage scenarios.

However, VLC channel modelling is a prerequisite for reliable VLC system design, which enables extensive utilization of Optical Wireless Communication (OWC) channel. Practical channel model construction requires knowledge of the channel statistical and physical parameters. Hence, channel simulations based on sole optical radiation patterns and received optical power is highly ambitious. Thereby, channel sounding, measurement of time-varying channel parameters is needed for robust real-world VLC system and VLC channel simulator design in addition to VLC channel model development.

The multipath OWC channel targeting VLC is known to exhibit low-pass behaviour due to LEDs frequency response. Furthermore, strong echo components are demonstrated to result with notches at certain frequencies [4]. To date, several studies have been conducted for infrared (IR) channel modeling [5]. However, considering the white LED light, covering a wide range of wavelengths with varying reflectivity, VLC channel modelling should be further expanded.

In the literature, several studies explored VLC channel modeling, as [6], [7] deducted root mean square (RMS) delay spread and path loss of VLC channel through Zemax raytracing software based simulations, [8] investigated indoor VLC channel with specular reflections using simulation environment. Furthermore, [9] studied VLC channel delay profiles for automotive applications utilizing LightTools software for ray-tracing simulations. Authors in [10] and [11] considered modelling of a geometry-based indoor VLC channel model. In [12] indoor VLC channel modelling through received optical power is conducted. [13]-[15] examined angular spread and path loss of V2LC channel lacking further characterization of small signal fading effects on V2LC channel. None of the studies to date, investigated outdoor VLC channel frequency and time domain characteristics such as Channel Frequency Response (CFR), power delay profile (PDP), RMS delay spread, mean excess delay with respect to varying distances, including LED and optical detector characteristics.

Channel sounding involves transmitting a known signal to excite the wireless channel for the purpose of acquiring chan- 
nel characteristics that can be described by the Channel Impulse Response (CIR) or CFR. Channel sounding techniques are classified into four, namely, time domain, frequency domain, spread spectrum sliding correlator based and compressed channel sounding. Time domain channel sounding involves transmission of a short duration pulse as an approximation of an impulse function, in order to receive its amplitude where a receiver measures the amplitude of the received signal. On the other hand, frequency domain sounding utilizes VNA to measure the wireless communication channel gain as $S_{21}$ parameters, across wide range of frequencies with great dynamic range. $S_{21}$ parameters yields, the complex amplitude of a sinusoidal stimulus transmitted from port 1 and received at port 2. VNA based frequency domain measurements further provide advantages such as noise immunity due to small receive filter bandwidth.

Furthermore, sliding correlation based sounder uses the correlation properties of two identical pseudorandom noise (PN) sequences due to the fact that the auto correlation of PN sequence resembles to auto-correlation of white noise, which is an impulse. Among the various channel sounding methodologies, VNA based static V2LC channel measurement is practical, as both receiver and transmitter circuits rely on same hardware clock for accurate synchronization. Moreover, high dynamic range of VNA enables efficient utilization of the APD dynamic range.

Considering VLC use case scenarios requiring short intervehicular distances such as stop and go traffic, vehicle to vehicle communications (V2V) between parked vehicles and fixed distance platooning applications, V2LC channel characterization is believed to be key to utilize the technology efficiently.

The goal of this paper is to characterize V2LC static channel, through VNA based frequency domain measurements. The original contributions of this paper is threefold. First, various automotive grade LED bulbs frequency domain characteristics are investigated and the effective usable bandwidth of each LED is determined. Second, best performing LED in terms of bandwidth performance and optical emitted power is selected. Furthermore, detailed frequency domain analysis of V2LC channel using automotive grade LED Day Time Running Light (DRL) is executed with respect to varying intervehicular distances, day light conditions including LoS and non-line-of-sight (NLoS) scenarios. Finally, end-to-end V2LC channel impulse response is extracted from frequency domain measurements to investigate time dispersion characteristics of the V2LC channel. To the best of our knowledge, this is the first work, conducting outdoor frequency domain V2LC channel measurements considering practical usage scenarios with typical VLC hardware constraints.

The rest of the paper is organized as follow. In Section II, we briefly describe the VNA based V2LC channel frequency sounder setup and in section III, we provide the employed methodologies for data processing for V2LC channel characterization. We further provide channel characteristics for static V2LC channel in Section IV. Finally, we conclude the paper in Section V.

\section{Measurement Setup}

In order to obtain the short range frequency responses of various automotive LEDs, Port 1 of Anritsu MS2026C VNA is feeded into the RF input of the bias tee, whereas the DC-bias selected with respect to the best obtained modulation bandwidth is feeded into the DC input of the bias tee. Hamamatsu S3884 - C5331 APD is attached to the port 2 of the VNA via $50 \Omega$ coaxial cable. Power amplifier at the output of port 1 and a low noise amplifier (LNA) at the receiving port 2 is utilized to have higher received power and therefore a higher signal to noise ratio (SNR). However, smaller receiver intermediate frequency (IF) filter $\left(\left(B_{I F}\right)\right)$ bandwidth to lower the noise can be also considered as an alternative in exchange to increased acquisition time. In order to characterize frequency response variation with respect to practical inter-vehicular distances, 2 low noise amplifiers are cascaded $(47 \mathrm{~dB})$ at the port 1 of the VNA before feeded into the RF port of the bias-tee. Required direct current (DC) bias is applied to the bias-tee in order to guarantee linear region working of LEDs.

At the receiver side APD output is amplified with a LNA (25 $\mathrm{dB}$ ) and feeded into the Port 2 of the VNA with the usage of 3 Huber-Suhner Sucoflex 404 shielded microwave cables with low insertion loss. $24 \mathrm{~m}$ of cable is used from APD output to VNA Port 2. Measurements are conducted from $100 \mathrm{kHz}$ to $10 \mathrm{Mhz}$, as the same calibration with all cables is utilized for all measurements with $N_{f}=4001$ points, averaged 5 sweeps. IF bandwidth $\left(B_{I F}\right)$ of $500 \mathrm{~Hz}$ is used to decrease the noise. Using $500 \mathrm{~Hz}$ of $\left(B_{I F}\right)$ leads a noise level equal to $-50 \mathrm{dBm}$ and an acquisition measurement time of $9.6 \mathrm{~s}$ for a single sweep.

In Fig.1 utilized channel sounding setup is demonstrated. At the transmitter side, LED DRL at the height of headlight is placed on a tripod, enabling elevation angle changes to characterize road reflections that can be encountered through different road inclinations. Utilized LED DRL consists of 4 independent LED, placed in 2 rows (See Fig.1.b).

At the receiver side, APD is placed with a $39^{\circ}$ angle which is same as the rear view camera of a production vehicle as demonstrated in Fig.2.c. Thereby, NLoS components reflected from road are targeted to be captured. Furthermore, placing the receiver with a certain inclination angle is found to be beneficiary in order to prevent APD saturation due to direct interaction with powerful vehicle headlights. APD incidence angle, elevation angle and height is not changed throughout the measurements, whereas its distance is changed for performance evaluations.

VNA is utilized both for transmitting and capturing channel sounding tones. Short, Open, Load, and Thru calibration is performed for the precision of measurements observing the device/ambient temperature changes for each run.

Frequency resolution $(\Delta f)$ obtained for measurements can be calculated as follows,

$$
\Delta f=\frac{f_{\max }-f_{\min }}{N_{f}-1}
$$




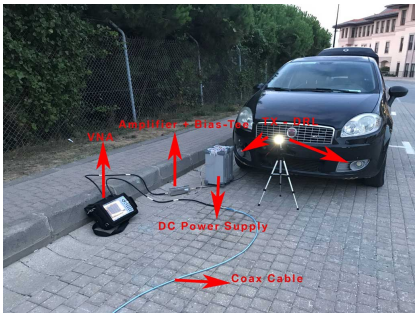

(a)

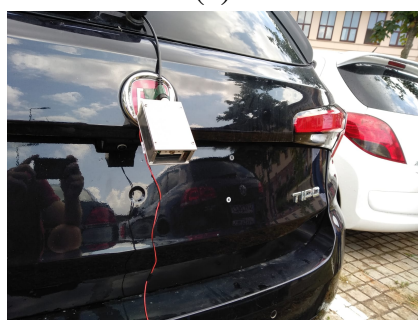

(c)

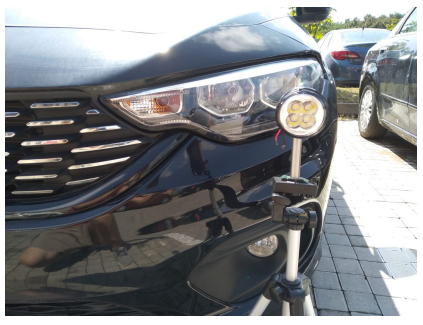

(b)

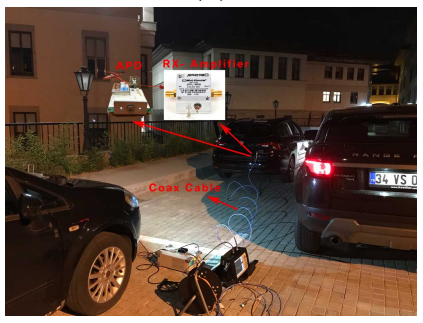

(d)
Figure 1: (a) Transmitter Measurement Setup (b) Transmitter DRL LED (c) Orientation of APD (d) Receiver Measurement Setup For NLoS Evaluation

where $f_{\min }$ and $f_{\max }$ are $100 \mathrm{khz}$ and $10 \mathrm{Mhz}$ respectively. Thereby, with a frequency step of $2.475 \mathrm{kHz}$, the frequency resolution further yields a maximum excess delay of 101 ns. Channel transfer functions are obtained through $S_{21}$ scattering parameter. The time-delay responses and power delay profile (PDP) are obtained from the complex channel transfer functions via the inverse Fourier transform.

Three different measurement campaigns namely sunny day, sunset and night are conducted in order to explore the effect of the lights. Measurement location $\left(41^{\circ} 12^{\prime} 28.4^{\prime \prime} \mathrm{N}\right.$ $\left.29^{\circ} 04^{\prime} 26.7^{\prime \prime} \mathrm{E}\right)$ is selected to be free of nearby objects. Sunny day measurements are conducted between 18.10-18.50 hours where the sun altitude angle varies between $13.25^{\circ}$ $20.72^{\circ}$ and azimuth angle changes from $271.93^{\circ}$ to $278.31^{\circ}$. On the other hand, sun altitude angle is recorded between $-3.09^{\circ}$ and $7.93^{\circ}$ for sunset measurements, whereas azimuth angles varied from $282.9^{\circ}$ to $293.17^{\circ}$. No optical filter is utilized to practically include the background lighting noise effect on the channel characterization. Furthermore, for night measurements street lights are also turned on for practical performance evaluation. APD aperture is kept in the opposite direction to direct sun for consistent measurement results, as direct sun light is observed to saturate APD. Optical spectrum of utilized LEDs is measured with Torus Toroidal Grating Spectrometer, in order to select the LED providing highest optical power between $400 \mathrm{~nm}-800 \mathrm{~nm}$ wavelengths, corresponding to the sensitive region of APD, for channel measurements. The measured data is further processed using MATLAB.

\begin{tabular}{ll}
\hline Parameter & Value \\
\hline Sweep Bandwidth & $9.9 \mathrm{MHz}$ \\
\hline Number of Points & 4001 \\
\hline IF BW & $500 \mathrm{~Hz}$ \\
\hline Acquisition Time (5 Sweeps) & $48 \mathrm{Seconds}$ \\
\hline RF Power & $22 \mathrm{dBm}(-25 \mathrm{dBm}$ VNA) \\
\hline
\end{tabular}

\section{Measurement Data Processing}

\section{A. Vehicle LEDs For VLC}

LEDs are mainly utilized for their long life time, faster response and small chip sizes enabling compact automotive light design for automobiles. Furthermore, automotive LED driving circuits are designed to provide both energy efficiency and dimming capability using pulse width modulation (PWM). However, none of the automotive LEDs to date, are designed solely for VLC purposes. Hence, we initially compared various automotive grade LED bulbs to decide about the best performing LED to further utilize for channel measurements. Fig.2 demonstrates that custom LED DRL offers the third highest bandwidth available within the compared LEDs. However, considering LED with the highest bandwidth to be a point source of red automotive tail-light LED (Lumileds Signal Sure 250) and the second highest bandwidth offering LED to be 3 chip automotive LED bulb (OSRAM LE UW U1A3) with no lens and reflectors, custom LED DRL with third highest available bandwidth providing highest optical power, enclosed in a casing with reflectors is selected for channel measurements (See Fig.3.).

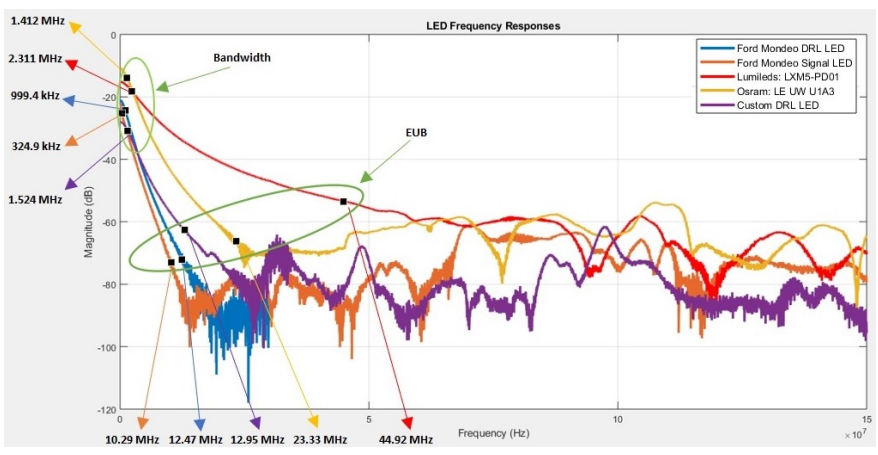

Figure 2: Bandwidth and EUB of automotive LEDs

In Fig. 2 it has been observed that in addition to $3 \mathrm{~dB}$ bandwidths, LEDs also provide almost linear frequency responses up to certain frequencies which we named as EUB. It has been concluded that, $30 \mathrm{~dB}$ bandwidth can be safely considered as EUB under ambient noise free conditions for many automotive LEDs when there is no OWC channel between LED and receiver APD. It should be noted that, as the LED optical output decreases at higher frequencies, EUB decreases with the increased inter-vehicular distance and day-light conditions due to limited dynamic range and sensitivity of the APD. Hence, knowing the frequency response characteristics of LED with inter-vehicular distance and day-light conditions, a power optimized waveform can be feeded into LED to obtain flat 
response up to the EUB. In Section IV, detailed EUB analysis of the selected LED will be provided.

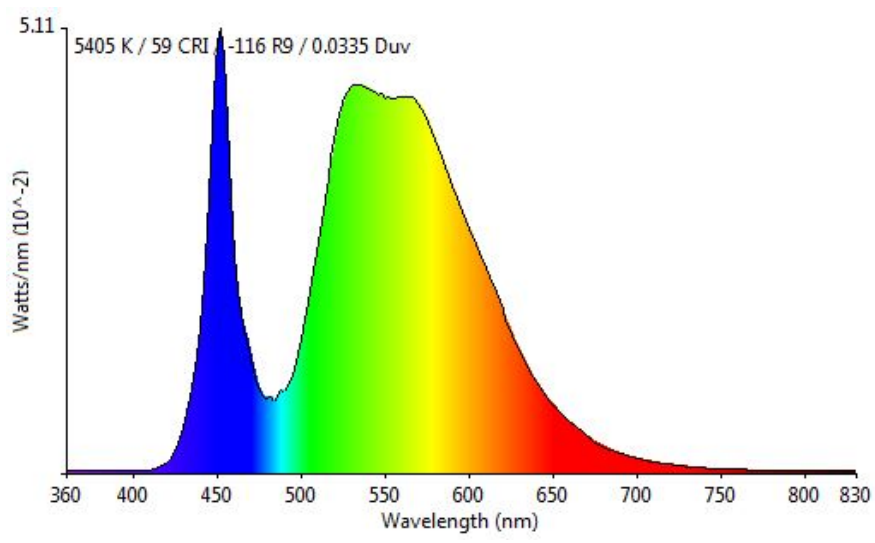

Figure 3: Automotive DRL LED Measured Spectrum

\section{B. LED Non Linear Characteristics}

Electrical-optical $3 \mathrm{~dB}$ bandwidth of LED simplified expression related to differential carrier lifetime is given by [16],

$$
f_{s}=\frac{1}{2 \pi} \sqrt{A^{2}+\frac{4 B}{q V} I}
$$

where A and B are Shockley-Read-Hall (SRH) and radiative recombination coefficients, I is the operation current, $\mathrm{V}$ is the operation voltage and $\mathrm{q}$ is the elementary charge. Thus, DCbias plays an important role to utilize the $3 \mathrm{~dB}$ bandwidth of LEDs. On the other hand, series connected LEDs are demonstrated to perform better in terms of V-I linear region characteristics due to the improved impedance, whereas parallel connected LEDs suffer from larger forward current thermal effect resulting non-linearity [17]. Thereby, custom automotive DRL made up from 4 series connected LEDs is preferred in addition to the other selection factors in Section II. Optimum DC-bias is determined through voltage sweep between $7 \mathrm{~V}$ to $11 \mathrm{~V}$ and modulation BW of the LED under consideration is observed to decrease above $10.8 \mathrm{~V}$ DC-bias due to internal quantum efficiency degradation. VNA RF output is amplified $47 \mathrm{~dB}$, feeding $22 \mathrm{dBm}$ to the LED to increase measurement signal tone level.

\section{Time Domain Analysis}

Frequency domain channel analysis quantified through the $S_{21}$ parameter measurements from $100 \mathrm{khz}$ to $10 \mathrm{Mhz}$. As both transmitter and receiver of the VNA are triggered with the same source, accurate timing information is obtained for measurements. Despite the VNA's and APDs higher bandwidth measurement capabilities, due to the negligible effect of LED on the channel frequency response above $10 \mathrm{Mhz}$, measurements are conducted up to $10 \mathrm{MHz}$.

Furthermore, frequency domain response is transformed into time domain via inverse discrete Fourier transform (IDFT). Denoting $S_{i j}\left(f_{n}\right)$ as the frequency domain $\mathrm{S}$ parameters, where $\mathrm{i}$ and $\mathrm{j}$ are the port indices and $f_{n}$ is the nth recorded frequency sample. Taking the IDFT, impulse response $h_{i j}$ is expressed as,

$$
h_{i j}\left(t_{n}\right)=\frac{1}{N_{f}} \sum_{k=0}^{N_{f}-1} S_{i j}(f n) e^{i 2 \pi k n / N_{f}}
$$

where $t_{n}$ is the $\mathrm{n}$ th time sample.

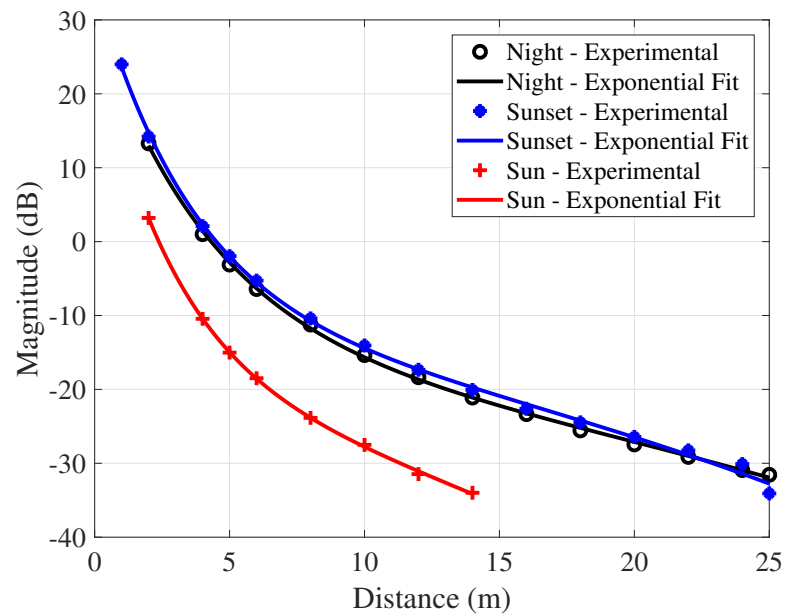

Figure 4: Direct LoS path loss under sunny, sunset and night conditions.

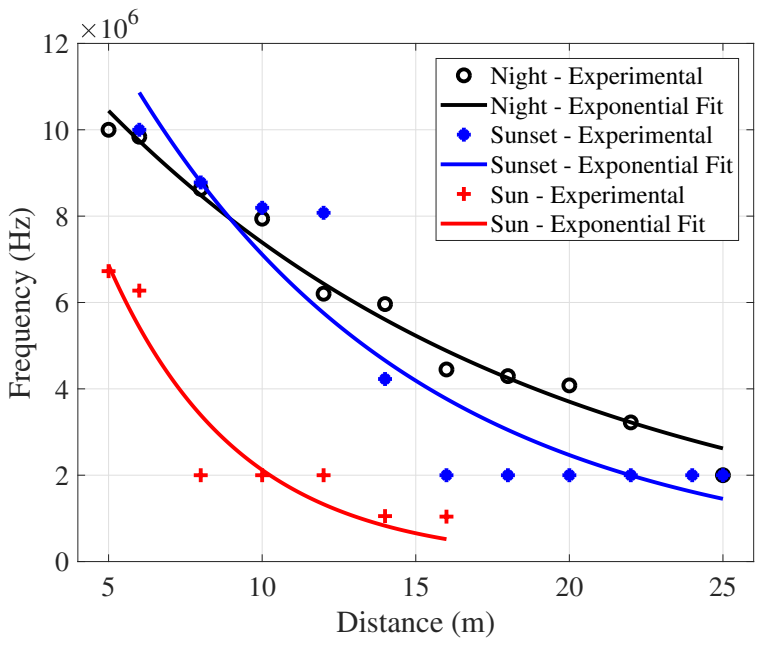

Figure 5: EUB w.r.t. distance under sunny, sunset and night conditions.

Time resolution in the time domain is determined through the measured bandwidth in the frequency domain. IDFT is calculated using the inverse fast fourier transform (IFFT). When using direct IFFT for the frequency domain samples measured by VNA, time resolution corresponds to $\delta t=\frac{1}{B_{\text {meas }}}$ where $B_{\text {meas }}$ is the measured bandwidth. In time domain, the number of points $\left(N_{f}\right)$ is the same as in frequency domain. Hence, in order to increase the time resolution from $101 \mathrm{~ns}$ to 

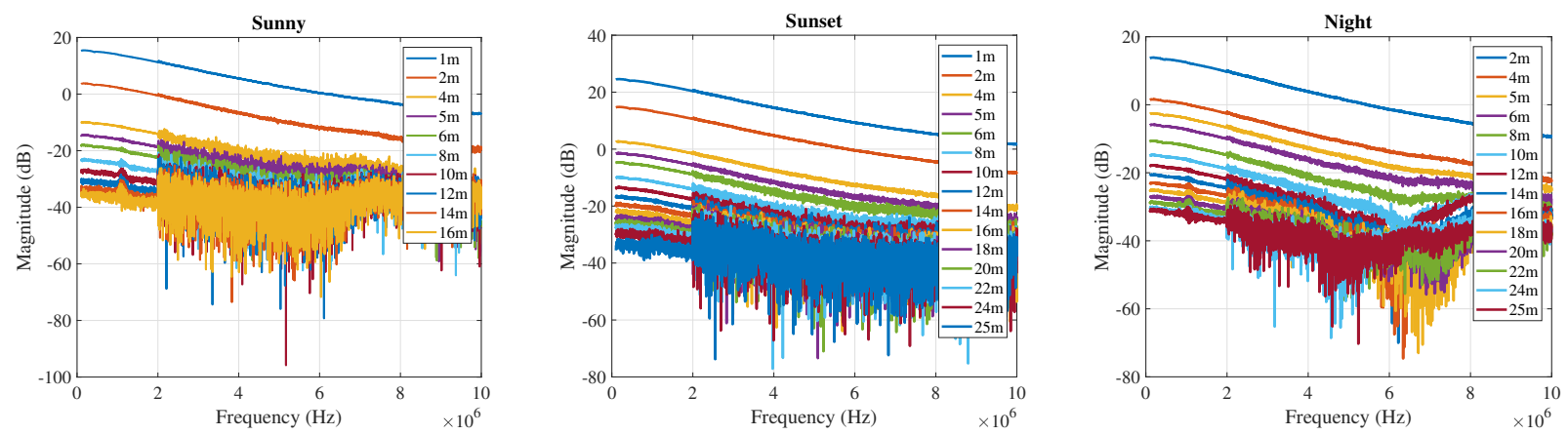

Figure 6: Direct LoS channel frequency response w.r.t distance.

$1 \mathrm{~ns}$, zero padding is performed before IFFT. Furthermore, considering the measured frequency response to be single sided spectrum, mirror of the measurement is also appended before the IFFT process. For analysis purposes, sampling frequency of $1 \mathrm{GHz}$ and Hanning IFFT window are utilized. Channel frequency response, including ambient and system noise, when LED is turned-off, is subtracted for more precise results. Considering zero padding, interpolation to for FFT size of 4096 with windowing steps to increase time resolution and avoid spectral leakage, of the obtained time domain response is re-calculated to check its consistency with the measured frequency responses. It has been observed that, due to windowing regenerated frequency response is slightly less powered than its measured counterpart. Hence, accuracy of our processing scheme is validated.

The noise floor power in the time domain $P_{n}$ is $-100 \mathrm{dBm}$, calculated from,

$$
P_{n}=\frac{\phi_{0} \cdot B_{I F}}{N_{f}}
$$

where $\phi_{0}$ is typical spectral power density of $-110 \mathrm{dBm} / \mathrm{Hz}$ considering for the used VNA. However, due to limited dynamic range of the APD, $-50 \mathrm{dBm}$ is the measurement noise floor.

The channel frequency response represents the channel behavior as a function of frequency. In the time domain, communication channel is characterized by its channel impulse response $h(t)$. The impulse response is the inverse Fourier transform of the frequency response, hence the received signal $y(t)$ in time domain is expressed with,

$$
y(t)=h(t) * x(t)
$$

where $x(t)$ is the transmitted signal and $(*)$ denotes the time domain convolution of the transmitted signals with the channel impulse response.

Considering multi-path components (MPCs) sourced from reflections of transmitted optical signals, PDP represents received powers at each time instant $\tau$ and written as,

$$
P D P(\tau)=|h(t)|^{2}
$$

Mean excess delay is described as first moment of the PDP, given by,

$$
\tau_{0}=\frac{\int_{0}^{\infty} t h(t) d t}{\int_{0}^{\infty} h(t) d t}
$$

RMS delay spread is defined as the square root of the second central moment of PDP and can be obtained from,

$$
\tau_{R M S}=\sqrt{\frac{\int_{0}^{\infty}\left(t-\tau_{0}\right)^{2} h(t) d t}{\int_{0}^{\infty} h(t) d t}}
$$

\section{V2LC CHANNEL CHARACTERIZATION}

V2LC channel is characterized through channel path loss, mean excess delay and RMS delay spread parameters. For direct $\operatorname{LoS}$ scenarios without any nearby objects, due to negligible MPC, road reflections are considered to explore time dispersion characteristics of the channel. On the other hand, nearby vehicle is also observed to cause constructive interference, thereby, increasing channel gain as depicted in Fig.7.

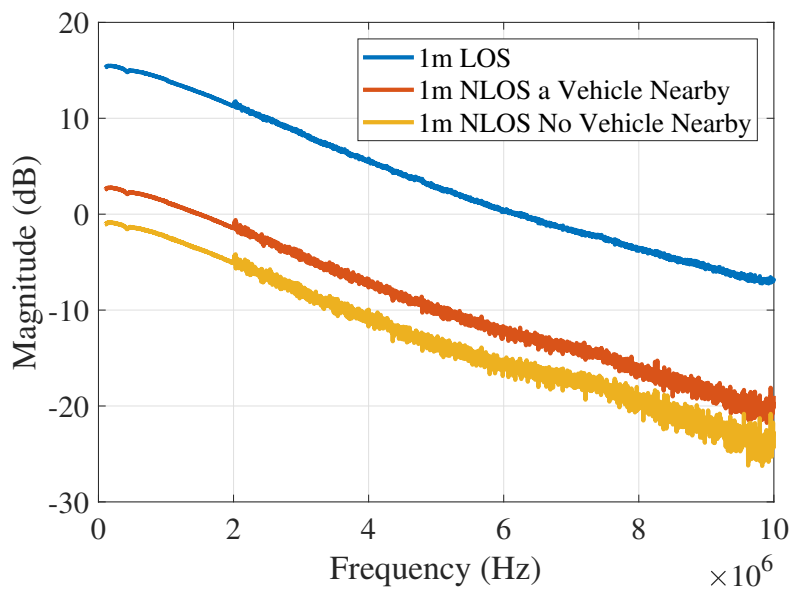

Figure 7: NLoS sunny condition. 


\section{A. Vehicular VLC Channel Path Loss}

Path loss has been characterized according to frequency response amplitudes for sunny day, sunset and night time measurements as depicted in Fig.6. $H_{0}$ expression for all usage scenarios are obtained via curve fitting. As measurements were conducted for $0^{\circ}$ incidence and radiance angles, with same transmitter-receiver heights, all path loss equations with respect to inter-vehicular distances are derived for direct $\operatorname{LoS}$ usage scenarios.

$H_{0}=\left\{\begin{array}{c}-20.11 e^{0.03924 d}+47.52 e^{-0.3245 d_{t x-r x}} \text { Sunny } \\ -11.74 e^{0.04112 d}+46.75 e^{-0.2654 d_{t x-r x}} \text { Sunset } \\ -15.1 e^{0.03016 d}+45.64 e^{-0.2259 d_{t x-r x}} \text { Night Time }\end{array}\right.$

It can be concluded that for same inter-vehicular distance, for instance at $14 \mathrm{~m}$, night VLC channel provides $12.943 \mathrm{~dB}$ more gain than sunny day time conditions yielding 5.963 Mhz, 1.053 Mhz EUBs respectively. However, night and sunset conditions are almost the same such that at $14 \mathrm{~m}$, the channel gain difference is $0.793 \mathrm{~dB}$ and the EUBs are 5.963 Mhz, 4.228 Mhz respectively. Thereby, providing an additional power in the amounts of channel losses with the consideration of preserving LED linear working conditions (i.e. optimization of DC-bias and RF power) can be utilized to enhance EUBs of LEDs at further distances.

However, considering increased inter-vehicular distances, nearby vehicles and varying day light conditions, EUB is observed to change. Fig.5 depicts the decrease of the EUB with respect to increasing inter-vehicular distance, and ambient light effect on EUB.

\section{B. Nearby Vehicle Effect on VLC Channel}

On the other hand, according to Fig. 7 it can be concluded that, a nearby vehicle located in the nearest lane, helps to increase the channel gain around $3.519 \mathrm{~dB}$ for day time, considering $1 \mathrm{~m}$ inter-vehicular distance scenario.

\section{Mean Excess Delay}

Mean excess delay $\left(\tau_{0}\right)$ is evaluated through night time measurements where LED DRL is intentionally directed towards the road to observe the effects of road and pavement reflections. $\tau_{0}$ for inter-vehicular distances from $2 m$ to $18 m$ is depicted on Table I. For all analysis, MPCs within $23.6 \mathrm{~dB}$ of the maximum are considered for 979 ns length as depicted on Fig.8. Mean excess delay is found to be increasing with the inter-vehicular distance.

$$
\tau_{0-f i t}=7.512 * 10^{-9} d+1.297 * 10^{-7} d
$$

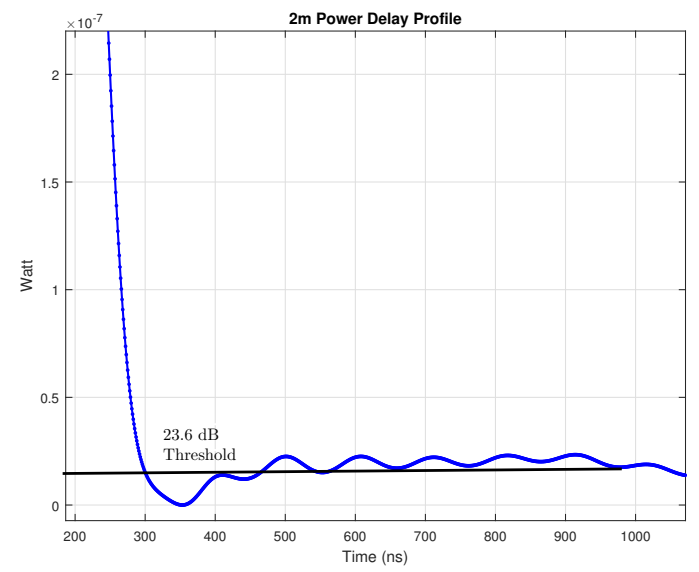

Figure 8: Maximum Excess Delay 23.62dB

\section{RMS Delay Spread}

RMS delay spread $\left(\tau_{R M S}\right)$ is observed to be correlated with inter vehicular distance as it increases with the transmitterreceiver distance. Both LoS and NLoS components are captured at the receiver. However, up to $10 \mathrm{~m} \mathrm{LoS} \mathrm{component} \mathrm{is}$ more stronger than the NLoS, where NLoS component appears to be within the LoS impulse response as depicted in Fig. 9.

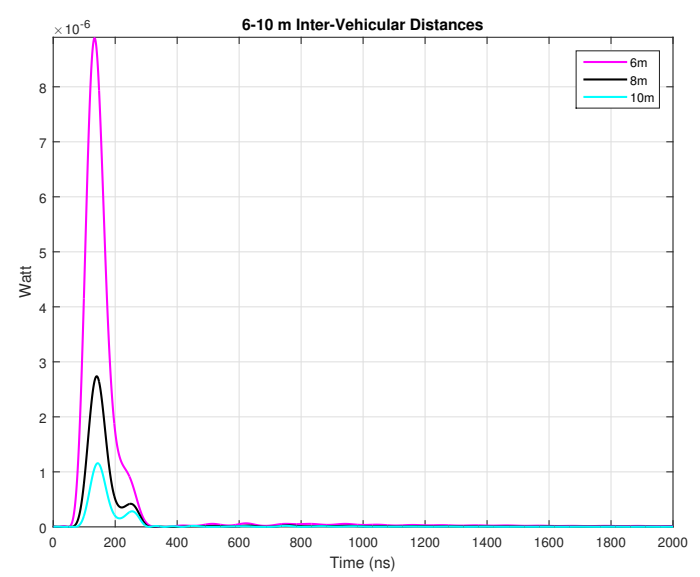

Figure 9: VLC Channel PDP for 6-10 m Inter-Vehicular Distance

Variations in the RMS delay spread for distances more than $6 m$ increases. Furthermore, with the increased inter-vehicular distance, road reflections start to become more obvious. Fig.10 depicts the road reflections mainly sourced by the bottom row LED of the LED DRL under consideration. At $18 m$ inter-vehicular distance NLoS component resulted from road reflections is observed to be in equal strength with the faded direct LoS light. This can be explained with the increased scattering of light (photons) at increased inter-vehicular distances. Furthermore, it can be concluded that, LED design plays an important role with the channel characteristics. If LED is either aligned to avoid road illumination or designed solely for LoS lighting, multipath components are expected to be very weak that may not have any significant effect on the 
channel time domain dispersion characteristics. However, on the other hand, as long as the vehicle LED illuminates road surface, reflections at longer distances should be taken into account to avoid inter-symbol interference (ISI).

$$
\tau_{R M S-f i t}=6.513 * 10^{-9} d+9.295 * 10^{-8} d
$$

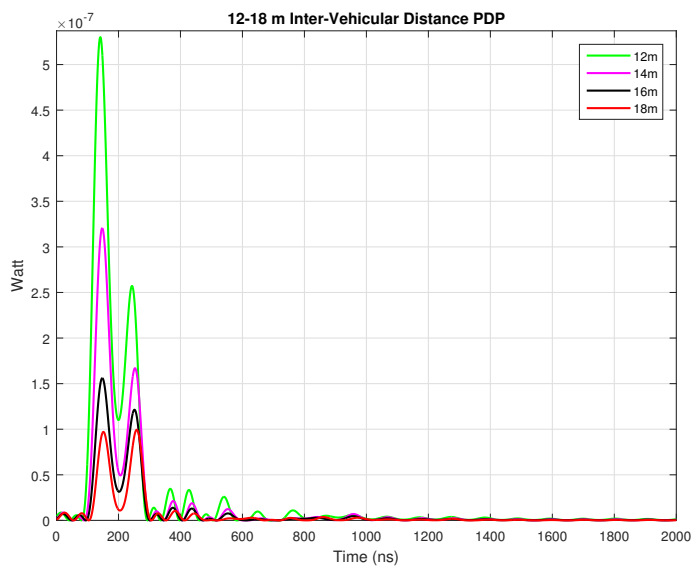

Figure 10: VLC Channel PDP for 12-18 m Inter-Vehicular Distance

Table I: Vehicular VLC Channel Characteristics

\begin{tabular}{|c|c|c|}
\hline Longitudinal Distance & $\tau_{0}(\mathrm{~ns})$ & $\tau_{R M S}(\mathrm{~ns})$ \\
\hline $2 \mathrm{~m}$ & 152.11 & 110.56 \\
\hline $4 \mathrm{~m}$ & 158.75 & 114.29 \\
\hline $6 \mathrm{~m}$ & 168.57 & 121.50 \\
\hline $8 \mathrm{~m}$ & 184.63 & 143.44 \\
\hline $10 \mathrm{~m}$ & 202.99 & 167.11 \\
\hline $12 \mathrm{~m}$ & 227.56 & 185.92 \\
\hline $14 \mathrm{~m}$ & 230.29 & 175.22 \\
\hline $16 \mathrm{~m}$ & 252.69 & 202.13 \\
\hline $18 \mathrm{~m}$ & 265.43 & 206.10 \\
\hline
\end{tabular}

\section{CONCLUSiON}

This work presents initial investigations of the V2LC channel characteristics using automotive LED light with a commercial VNA. Measurement results indicate that, LEDs $30 \mathrm{~dB}$ modulation bandwidths can be utilized for practical inter-vehicular communication scenarios, using vehicle power LED. Unlike RF power, optical power at the higher modulation frequencies can be increased up to DC level enabling higher modulation bandwidth usage of LEDs. Furthermore, EUB of a V2LC system is observed to changes with the ambient light conditions and inter-vehicular distances, due to limited dynamic range of photo-detectors (PDs). However, considering new generation of vehicles equipped with range finding sensors (radar, lidar, camera) and ambient light sensors (sun load, rain, light sensor), knowing inter-vehicular distances and ambient light conditions, adaptive bandwidth usage of LED will be possible, providing efficient end-to-end V2LC system bandwidth utilization.
Furthermore, as LED modulation bandwidth also depends on the bias voltage, optimum operating voltage should be utilized for best communication performance and precise channel sounding.

V2LC channel is known to exhibit frequency flat characteristics. However, LED modulation bandwidth and reflections from nearby objects are also demonstrated to play an important role for the frequency response and time dispersion characteristics of the V2LC channel. Nearby vehicles are demonstrated to result with constructive interference, which increases channel gain.

On the other hand, our V2LC channel measurements depicts that, with the increased inter-vehicular distances, NLoS components from road reflections become more comparable to $\mathrm{LoS}$ component. At $18 \mathrm{~m}$ inter-vehicular distance, NLoS components from road reflections are observed to provide same optical power as the LoS components at the APD. Moreover, RMS delay spread is depicted to be correlated with the inter-vehicular distance, increasing with the distance for the measurements conducted.

Sun light is observed to decrease the system EUB as the APD has a limited dynamic range and DC light from sun increases the noise floor of the system. Under sunny day light conditions, transmitted signal can be consistently detected up to $16 \mathrm{~m}$ whereas at sunset and night conditions detection range extends beyond $25 \mathrm{~m}$. Hence, it can be concluded that, sun decreases the maximum data transfer distance range when no optical filter at the receiver is used.

\section{REFERENCES}

[1] A. T. Hussein, M. T. Alresheedi, and J. M. Elmirghani, "20 gb/s mobile indoor visible light communication system employing beam steering and computer generated holograms," Journal of lightwave technology, vol. 33, no. 24, pp. 5242-5260, 2015.

[2] D. Karunatilaka, F. Zafar, V. Kalavally, and R. Parthiban, "Led based indoor visible light communications: State of the art.," IEEE communications surveys and tutorials, vol. 17, no. 3, pp. 1649-1678, 2015.

[3] W.-H. Shen and H.-M. Tsai, "Testing vehicle-to-vehicle visible light communications in real-world driving scenarios," in Vehicular Networking Conference (VNC), 2017 IEEE, pp. 187-194, IEEE, 2017.

[4] J. M. Kahn and J. R. Barry, "Wireless infrared communications," Proceedings of the IEEE, vol. 85, no. 2, pp. 265-298, 1997.

[5] H. Schulze, "Frequency-domain simulation of the indoor wireless optical communication channel," IEEE Transactions on Communications, vol. 64, no. 6, pp. 2551-2562, 2016.

[6] F. Miramirkhani, O. Narmanlioglu, M. Uysal, and E. Panayirci, "A mobile channel model for vlc and application to adaptive system design," IEEE Communications Letters, vol. 21, no. 5, pp. 1035-1038, 2017.

[7] F. Miramirkhani and M. Uysal, "Channel modeling and characterization for visible light communications," IEEE Photonics Journal, vol. 7, no. 6, pp. 1-16, 2015.

[8] J. Chen and C. Yan, "A channel model for indoor visible light communication system with specular reflection," in Optical Communications and Networks (ICOCN), 2017 16th International Conference on, pp. 1-3, IEEE, 2017.

[9] S. Lee, J. K. Kwon, S.-Y. Jung, and Y.-H. Kwon, "Evaluation of visible light communication channel delay profiles for automotive applications," EURASIP journal on Wireless Communications and Networking, vol. 2012, no. 1, p. 370, 2012.

[10] Y. Qiu, H.-H. Chen, and W.-X. Meng, "Channel modeling for visible light communicationsa survey," Wireless Communications and Mobile Computing, vol. 16, no. 14, 2016. 
[11] A. Al-Kinani, C.-X. Wang, H. Haas, and Y. Yang, "A geometry-based multiple bounce model for visible light communication channels," in Wireless Communications and Mobile Computing Conference (IWCMC), 2016 International, pp. 31-37, IEEE, 2016.

[12] L. Mucchi, F. S. Cataliotti, L. Ronga, S. Caputo, and P. Marcocci, "Experimental-based propagation model for vlc," in Networks and Communications (EuCNC), 2017 European Conference on, pp. 1-5, IEEE, 2017.

[13] W. Viriyasitavat, S.-H. Yu, and H.-M. Tsai, "Short paper: Channel model for visible light communications using off-the-shelf scooter taillight," in Vehicular Networking Conference (VNC), 2013 IEEE, pp. 170-173, IEEE, 2013.

[14] A.-L. Chen, H.-P. Wu, Y.-L. Wei, and H.-M. Tsai, "Time variation in vehicle-to-vehicle visible light communication channels," in Vehicular Networking Conference (VNC), 2016 IEEE, pp. 1-8, IEEE, 2016.

[15] Z. Cui, C. Wang, and H.-M. Tsai, "Characterizing channel fading in vehicular visible light communications with video data.," in $V N C$, pp. 226-229, 2014.

[16] P. Deng and M. Kavehrad, "Effect of white led dc-bias on modulation speed for visible light communications," arXiv preprint arXiv:1612.08477, 2016.

[17] P. Deng, M. Kavehrad, and M. A. Kashani, "Nonlinear modulation characteristics of white leds in visible light communications," in Optical Fiber Communication Conference, pp. W2A-64, Optical Society of America, 2015. 